\title{
FRACTOGRAPHY ANALYSIS OF SUPPORTING ELEMENTS FRACTURED UNDER OPERATING CONDITIONS
}

\author{
VANYA DYAKOVA \\ Institute of Metal Science, Equipment and Technologies \\ with Hydro- and Aerodynamics Centre "Acad. A. Balevski", \\ Bulgarian Academy of Sciences, \\ e-mail:v_diakova@ims.bas.bg
}

\begin{abstract}
In the present paper a fractography analysis is performed for two supporting elements fractured under operating conditions at different loadings - cyclically and combined by pressure and torsion. The reasons for the destruction are stated. The fracture stages are determined, as well the zones of fatigue, brittle, ductile and atypical fractures and their elements are illustrated. The study is performed by means of the methods of light and scanning electron microscopy. The results are theoretically explained. Keywords: fractography, fatigue, ductile, brittle fracture, striations, beach marks, flutes, facets.
\end{abstract}

\section{INTRODUCTION}

Determination of the manner and causes of fracture of various equipment parts under operating conditions is a complex process that includes observation of the fractured elements and surfaces, as well as explaining the mechanism and causes of destruction. It is based on different methods of observation and analysis and uses a variety of techniques to apply them.

Both the transmission electron microscopie and scanning electron microscopie and their widespread use provide vast amounts of new information regarding the micromechanisms of fracture processes and make fractography an indispensable tool in failure analysis.

The micromechanism of ductile fracture, i.e. the initiation, growth, and coalescence of microvoids has been confirmed, and correlations between void (dimple) size/shape, stress state, and material cleanliness have been developed. Different models of explaining the mechanisms of fatigue fracture have been evolved, and correlations between fatigue striations, load cycles, striation spacing, and loading conditions have been developed [1].

DOI: 10.7546/EngSci.LVIII.21.03.06 
Conclusive experimental evidence regarding initiation mechanisms of fatigue fracture has also been acquired from electron fractogaphy studies [1-2].

In brittle fracture, explanations have been offered for the cleavage patterns that occur on fractured surfaces, and the form of the patterns has successfully been used to determine fracture direction and initiation points [2].

The aim of this paper is to explain the mechanism and to indicate the reasons for fracture of two supporting elements loaded by cyclic and combined loading of pressure and torsion under operating conditions.

\section{FRACTURE AS A RESULT OF CYCLIC LOADING}

A fracture that is the result of repetitive or cyclic loading is known as a fatigue fracture. Fatigue fractures are characterized by the existence on their surface of fatigue zones showing the stages of fatigue failure, Fig. 1. In the most general case, the process of fatigue crack development includes: (i) a stage of slow stable crack growth (zone Ls), (ii) a stage of accelerated unstable crack development (zone Lr), and (iii) a stage of static final fracture (Ld). Static final fracture occurs when the length of the fatigue crack reaches the critical value $\mathrm{Lf}(\mathrm{Lf}=\mathrm{Ls}+\mathrm{Lr})$. Zone $\mathrm{Lf}$ is a zone of fatigue crack development [3]. The type and size of the fatigue zones depend primarily on the type of the material and on the number of the load cycles that the detail has undergone. The shape of the fatigue zones depends on the shape of the detail and on the scheme of the applied stress.

The main elements of the morphology of the fatigue fracture surface are: beach marks, fatigue striations and secondary fatigue cracks.

Beach marks or clam shell are the most distinct characteristic of fatigue failures in the field markings on the cyclically grown portion of the fracture [1-2]. Similar marks on fractures can be produced under certain conditions by other fracture mechanisms that involve cyclic crack growth without cyclic loading. Also, such marks may not be visible on all materials that fail by fatigue; for example, many cast irons do not develop beach marks.

Fatigue striations are observed on the surfaces of many fatigue fractures of metals. They are more easily observed on the surfaces of more ductile metals, especially Face-Centered Cubic (FCC) metals, than on steels. Striations on low-strength steels tend to be wavy in nature and exist in patches rather than over the entire surface. Fatigue striations may be quite difficult to observe on high-strength steel fatigue fractures, depending on the manner of loading, the environment, and so on [1-2]. Striations are a visual record of the position of the fatigue crack front during crack propagation through the material.

Engineering Sciences, LVIII, 2021, No. 3 
According to the modern models, fatigue striations occur as a result of successive expansion and contraction of the side surfaces of the crack. When, due to tensile stresses, the mouth of the crack expands, the crack moves forward. In double-sided compression, the plastically deformed metal at the top of the crack undergoes flattening, which subsequently manifests itself in the form of parallel fatigue striations [3].

Secondary cracks are perpendicular to the primary fracture. The splitting appears when materials are broken by bending loads. The visual macro analysis often reveals secondary cracks that have propagated only partially through a cracked member $[1-2,4]$.

In our case, a torn ball joint bolt, part of a spring suspension of a freight wagon was examined The bolt has been subjected to cyclic loading under operating conditions of the wagon for more than ten years.

For the purposes of the fracture analysis, the chemical composition of the bolt steel was determined in advance, its microstructure was studied and the Brinell hardness was measured. It was found that the material of the fractured bolt is low-alloy steel C45, according to BSS EN 10027-1. Metallographic studies show that the microstructure of the steel is perlite-ferrite, as the ratio between the amounts of perlite to ferrite is 65:35.

Macrofractography observations of the fractured bolt surface show that the destruction of the hinge occurs perpendicular to its axis, Fig. 2. No macroplastic deformation of the fracture was observed. The destroyed surface is slightly oxidized.

For the purposes of the examination, a sample containing the fractured surface was cut from the object, Fig. 2. A large fatigue zone Ls is clearly

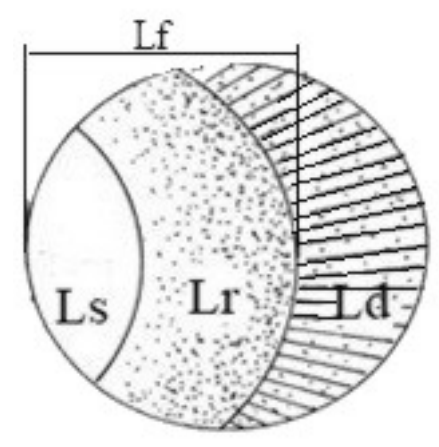

Fig. 1. Fatigue fracture structure diagram - zones Ls-Lr-Ld-Lf

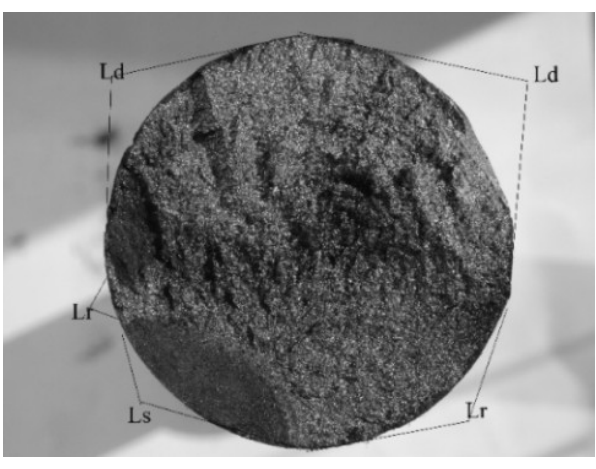

Fig. 2. General appearance of the fractured bolt surface 


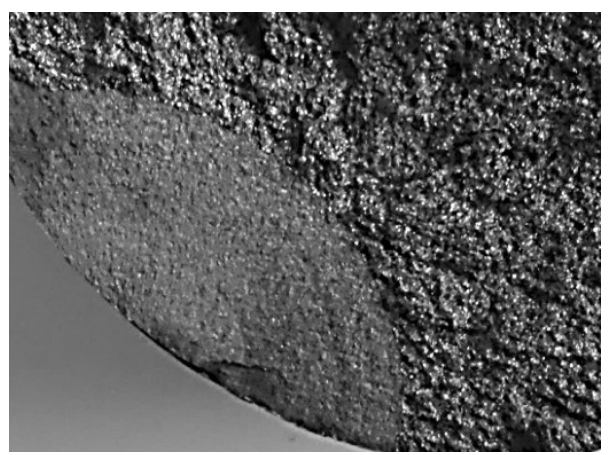

Fig. 3. Beach marks in zone Ls

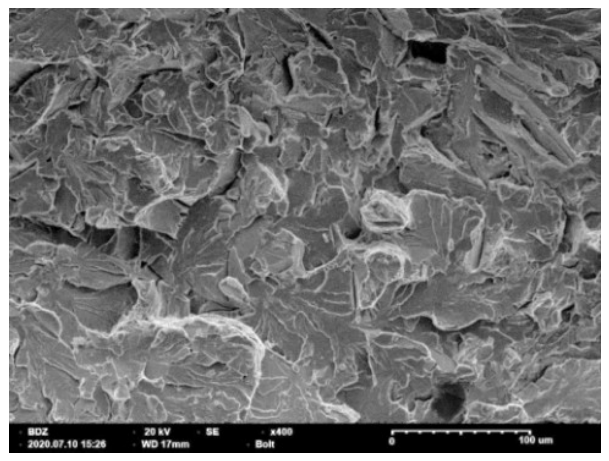

Fig. 5. Brittle fracture zone Lr

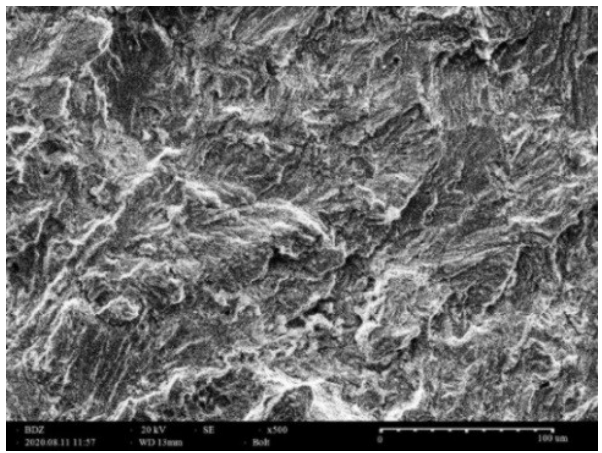

Fig. 4. Fatigue fracture zone Ls

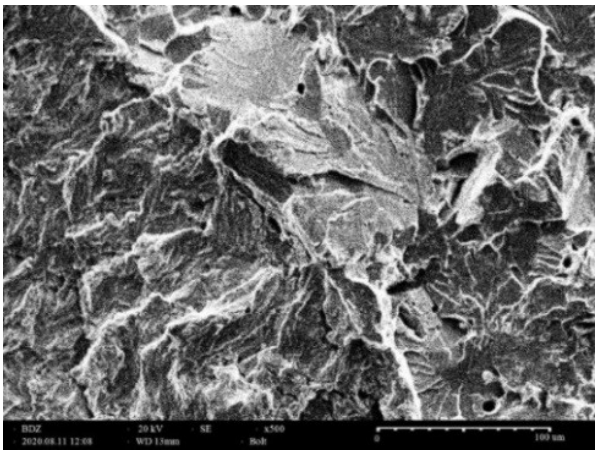

Fig. 6. Boundary between zone Ls and zone $\mathrm{Lr}$

visible on the surface. The photos show that the propagation of the fatigue crack started from the outer edge of the destroyed bolt surface.

The zone of slow stable growth of the crack Ls has a relatively smooth relief, Figs 2 and 3. There are clear "beach" marks, which are characteristic of fatigue fractures. The morphology of the fatigue zone observed with a Scanning Electron Microscope (SEM) is shown in Fig. 4. The relief is relatively weak, without a clear structure. No fatigue striations were observed, which probably were erased from the corrosion products during the corrosion process.

The zone $\mathrm{Lr}$ is formed due to the development of fatigue fracture. The bearing section of the bolt gradually decreases and accelerates the spread of the crack by a brittle mechanism, Fig. 2. In this zone the relief of the fractured surface is crystallographically pronounced - facets, characteristic of transcrystalline quasi-cleavage are observed, Fig. 5. Figure 6 shows the sharp boundary between the zones Ls and Lr. 


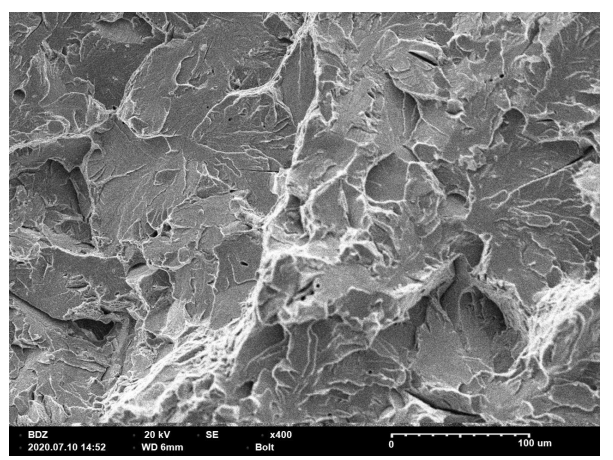

Fig. 7. Brittle fracture Zone Ld

The zone of static final failure Ld is formed after complete exhaustion of the bearing capacity of the bolt, Fig. 7. The relief of the destroyed surface is significantly rougher than in zone Lr. Elongated hills oriented in the direction of crack propagation are observed, Fig. 2. The fracture is performed by a brittle mechanism by quasi-cleavage.

No elements of inter-crystalline destruction were observed in any of the studied fractured surfaces. It was found that the percentage ratio between the surface with fatigue fracture Lf and that with accelerated fracture Ld is 46:54 according to the methods of quantitative metallography.

\section{Analysis of the observation results}

In the considered example above, the fracture of the bolt starts by a fatigue mechanism in a place located on its outer surface, and the most probable cause of the crack is the presence of defects on the surface of the bolt, which play the role of stress concentrators. Evidence that the fracture of the hinge begins gradually through a fatigue mechanism is the relatively smooth surface of the fracture around the original hearth, the presence of concentric fatigue beach marks and corrosion products over them.

Due to the reduction of the bearing section and the critical reduction of the bearing capacity of the bolt and under the influence of the cyclic loading to which it was subjected under operating conditions, the fracture continued by accelerated propagation of the crack by a brittle mechanism. This led to its complete fracture.

The brittle fracture is usually performed under the influence of normal stresses and spreads on the least packed crystallographic plane - cleavage plane [2]. It starts suddenly, spreads at high speed and low energy loss. In 
many cases, it leads to catastrophic destruction of structures under operating conditions.

\section{FRACTURE AS A RESULT OF PRESSURE AND TORSION}

Very often in the practice, the destruction of units and details is as a consequence of combining of several types of loading - pressure and torsion, torsion and tension, torsion and bending. As a result of them, some fractures have a unique appearance, such as quasi-cleavage and flutes and cannot be readily placed within any of the principal fracture modes.

Quasi-cleavage fracture is often a localized (isolated) feature on a fractured surface that exhibits characteristics of both cleavage and plastic deformation. The term quasi-cleavage does not accurately describe the fracture, as it suggests that the fracture is similar, but not cleavage. The term was coined because, although the central facets of a quasi-cleavage fracture strongly resembled cleavage, their identity as cleavage planes was not established until well after the term had gained widespread acceptance $[1,4]$.

Flutes exhibit elongated grooves or voids that connect widely spaced cleavage planes. The fracture process is known as fluting. The term flutes was apparently chosen because the fractures often resemble the long, parallel grooves on architectural columns or the pleats in drapes [1-4].

The second object we studied is a destroyed unit of a sensor device. The fracture of the unit occurred during the manually screwing a nut to the mounting stud in order to attach the unit to the main structure.

There is no data on the steel grade of the unit and its determination was not possible as the unit could not be further destroyed. The unit has not worked so far, i.e. it has not been subjected to fatigue load or vibrations. During the tightening of the nut, the unit has been subjected to pressure and torsion.

The destroyed unit consists of two parts, the first of which is of such dimensions that did not allow its fractured surface F1 to be observed with a microscope. The morphology of the fractured surface F2 of the stud, which is the second part of the unit, was studied using Light Microscope (LM) and SEM.

Macro-fractographic observations of F2 show that the fracture of the stud is in the plane perpendicular to its axis, where the chamfer and the channel are at the end of the stud, Fig. 8. There the cross-section of the stud is the smallest. No macro-deformation of the stud is observed.

Engineering Sciences, LVIII, 2021, No. 3 

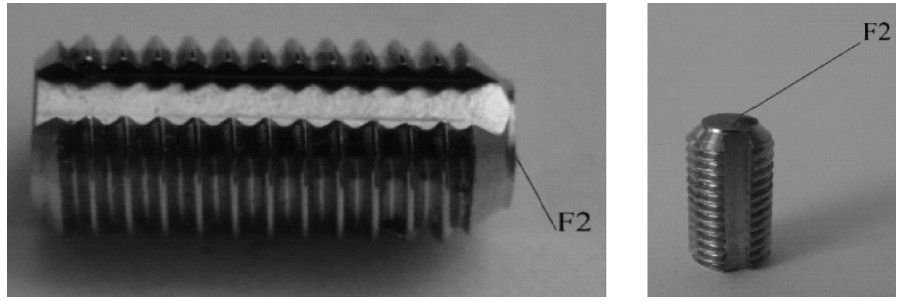

Fig. 8. Stud - destroyed surface F2

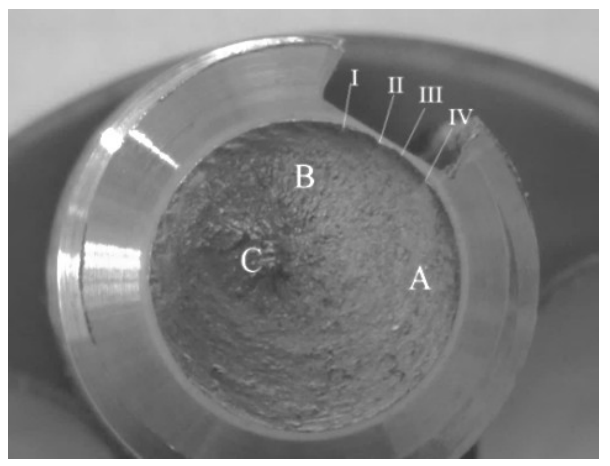

Fig. 9. Fractured surface F2

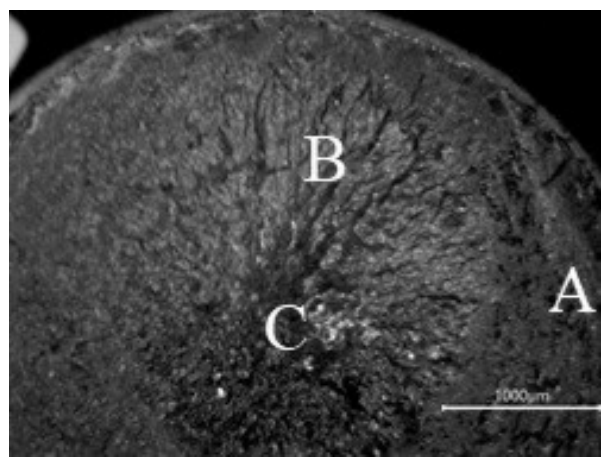

Fig. 10. Zones A, B and C

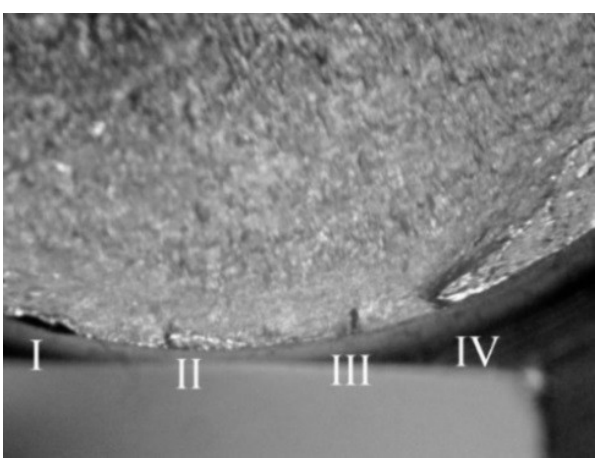

Fig. 11. Deformed sections on the outer edge of the stud 
Three zones marked as A, B and C, each of which has a different morphology of fractured surfaces can be seen in F2, Figs 9 and 10. Four micro-deformed sections marked $\mathrm{I} \div \mathrm{IV}$ are well distinguished on the outer edge of the stud, Figs 9 and 11.

Figure 12(a, b) illustrates the first and second deformed section I, II, respectively. The third section (III) is sharp, perpendicular to the outer edge of the stud, and the fourth section is the largest Fig. 12(c), so it is photographed with a stereo microscope. The sections are located very close to each other below the stud channel, where zone A is narrowest. In sections I and II, traces of rubbing are shown directed at an angle of 90 degrees to the outer edge of the stud in the first section, Fig. 12(a), and at an angle of 45 degrees to the outer edge in the second section, Fig. 12(b). The deformed sections finish in well-formed wide elongated zones with a very smooth surface,

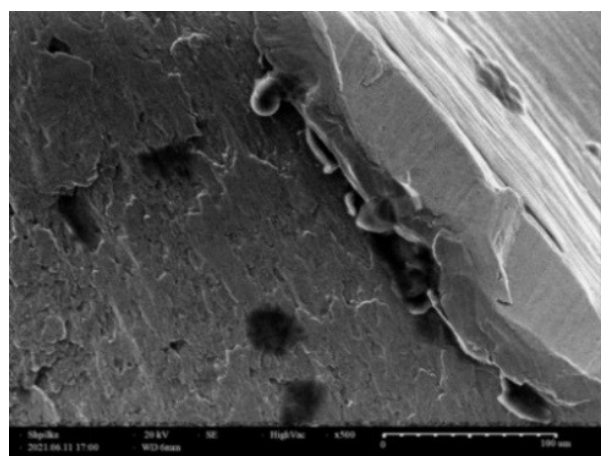

(a)

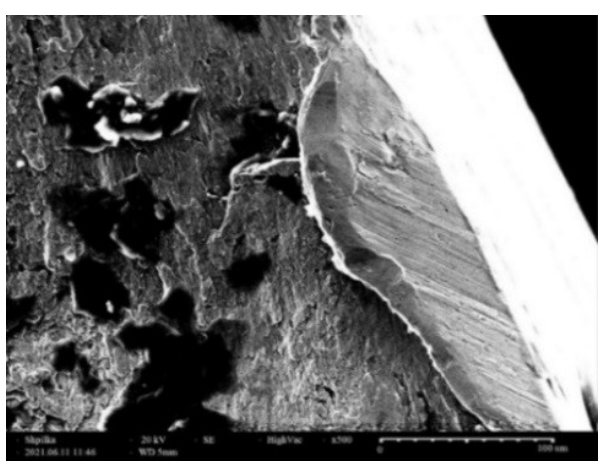

(b)

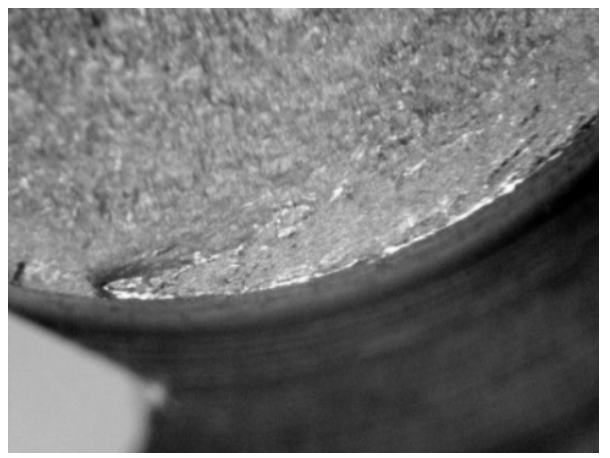

(c)

Fig. 12. Deformed sections on the outer edge of the stud: (a) section I; (b) section II; (c) sections III and IV 
which differ sharply from the surface morphology of zone A. The fourth section is a wedge-shaped and gradually expands. This is the beginning of zone A.

The morphology of zone A is shown in Figs $10 \div 15$. It has a relatively smooth relief, Fig. 13(a), the orientation of the structure completely follows the outer edge of the stud, Figs 13(b) and 14(a). The width of zone A is different in different areas. There are pores of different sizes, Figs $12 \div 15$, and traces of rubbing, which are parallel to the outer edge of the stud, Figs 14(b) and 15 .

The relief of zone B is very pronounced, Figs $10,14 \div 17$ and $19 \div 20$. There are pores, Fig. 14(a), and radially oriented hills and flutes formed by the fusion of the pores, Fig. 14 (b). The flutes pass through zone A only in single

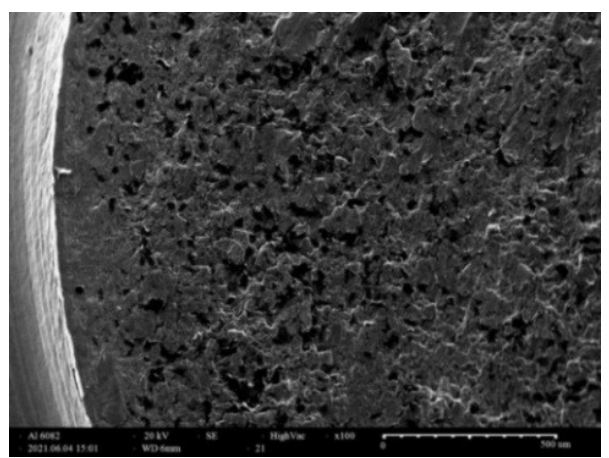

(a)

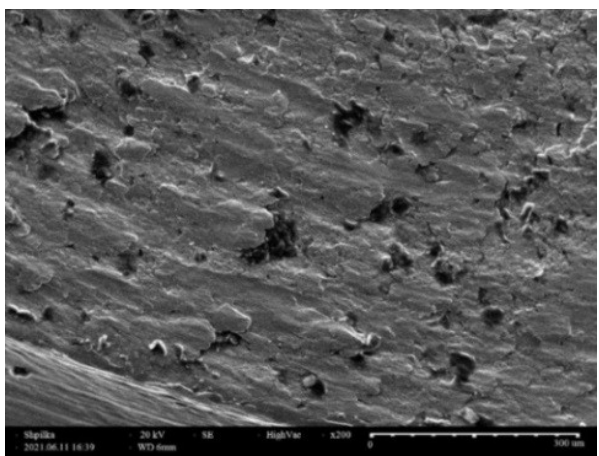

(b)

Fig. 13. Zone A

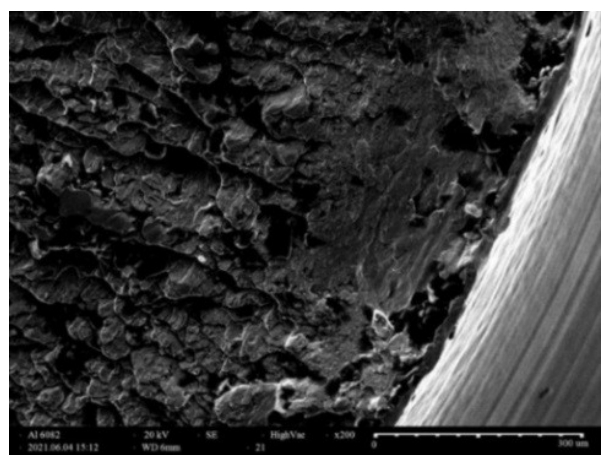

(a)

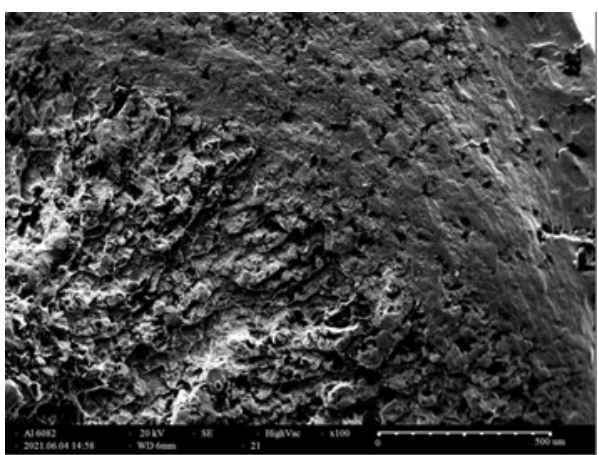

(b)

Fig. 14. Zone A and the beginning of zone B with well-defined pore-forming flutes 


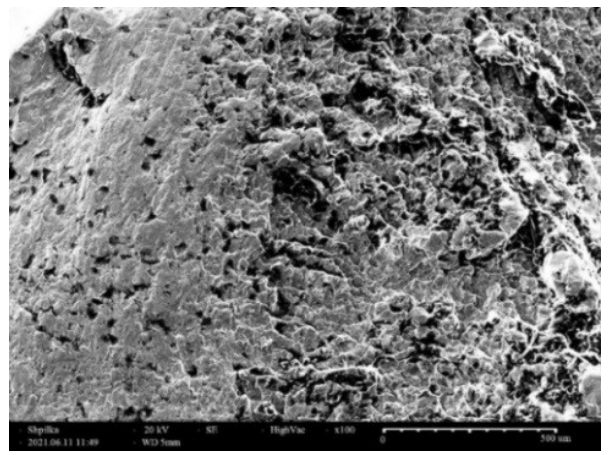

Fig. 15. Zone A, zone B with radially directed hills and flutes, and beginning of zone $\mathrm{C}$

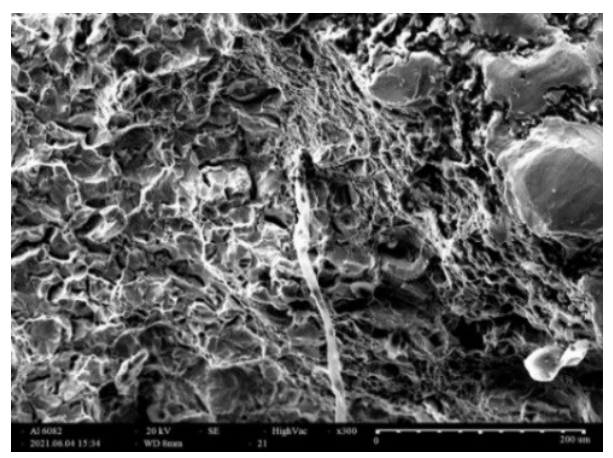

Fig. 17. Ductile fracture areas between zones $\mathrm{B}$ and $\mathrm{C}$

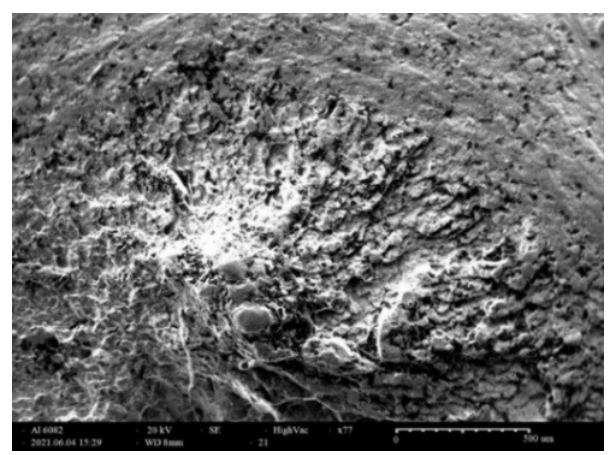

Fig. 19. Zone $\mathrm{C}$, radial flutes of zone $\mathrm{B}$ and zone $\mathrm{A}$

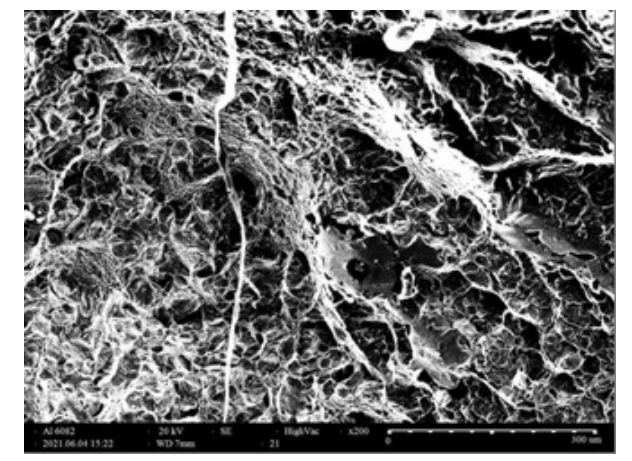

Fig. 16. Transition zone B to zone $\mathrm{C}$ - areas with ductile destruction

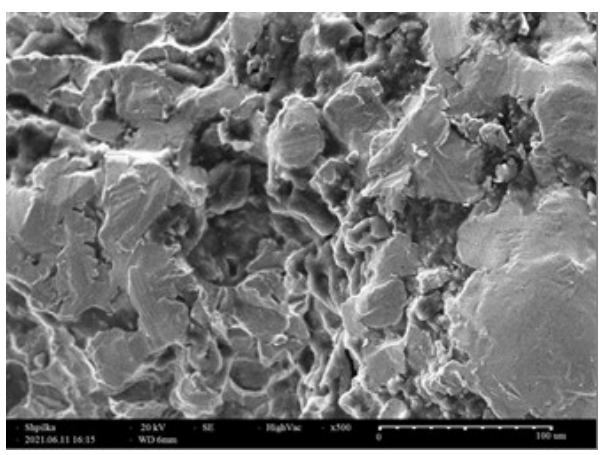

Fig. 18. Zone $\mathrm{C}$ and areas with brittle fracture around $\mathrm{C}$

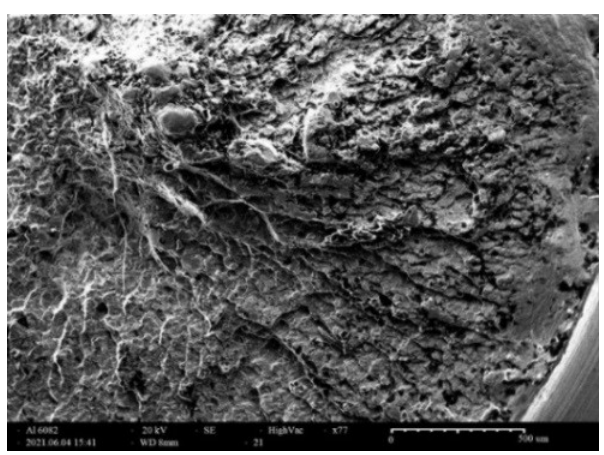

Fig. 20. Zones A, B and C

Engineering Sciences, LVIII, 2021, No. 3 
cases and reach the outer edge of the stud, Fig. 10. Figures $14 \div 15$ clearly show the transition between zones A and B. Areas with ductile fracture are observed in the transition zone between B and C, Figs 6 and 17 .

Zone $\mathrm{C}$ is close to the geometric axis of the stud, Figs 9 and 10, and it is lighter on the photos, Figs 19 and 20. Here several small areas with dimensions between $50 \mu \mathrm{m}$ and $100 \mu \mathrm{m}$ with flat surfaces are observed, Figs $17 \div 20$, and traces from rubbing, Fig. 18. There are both areas with ductile fracture, Figs 17, 19-20, and areas resembling brittle fracture, Figs 18-20, around the areas with atypical flat surface.

There are no elements of fatigue fracture - fatigue striations and "beach" marks in all three zones $\mathrm{A}, \mathrm{B}$ and $\mathrm{C}$.

The research and analysis in this publication were performed in the laboratories of IMSETHAC- BAS. All photos are taken by the author.

\section{Analysis of the observation results}

The fracture of the stud probably starts due to pressure and torsional stresses at the points located on the outer surface of the cylindrical part of the stud in the narrowest section, where the channel and the chamfer finish. These are the deformed sections shown in Fig. 12. It is possible that the microdeformations in these places are the result of pressure by the impact wrench and torsional shear in the direction of rotation when turning the nut. However, it is possible that there was a cut or other defect obtained by machining during the cutting of the channel or during the formation of the bevel of the stud. The defect is a stress concentrator and it is possible that the deformation and fracture of the stud have started due to the combination of compressive and torsions stresses. Because of the fracture, the bearing section of the stud has decreased and the accelerated propagation of the crack by a brittle mechanism has begun - zone B.

Finally, the stud is completely destroyed and detached from the unit. The zone $\mathrm{C}$ has been formed near the axis of the stud. Areas with a brittle transcrystalline fracture and areas with a ductile fracture are observed.

\section{REFERENCES}

[1] Failure Analysis and Prevention, ASM HANDBOOK (2002) 11.

[2] Fractography, ASM HANDBOOK (1987) 12.

[3] L. Engel and G. Klingele, Scanning Electron Microscopy, Fracture, Handbook, Metallurgy (1986) (in Russian). 
[4] G. A. Pantazopoulos, Short Review on Fracture Mechanisms of Mechanical Components Operated under Industrial Process Conditions: Fractographic Analysis and Selected Prevention Strategies, Metals (2019) 9 148, DOI: $10.3390 /$ met9020148.

Received June 25, 2021 